\title{
Constraining GW signatures in pulsar timing: A multi-frequency timing analysis
}

\author{
Jacques Maritz ${ }^{* \dagger}$ \\ Physics Department, University of the Free State, 9300, South Africa \\ E-mail: maritzjmeufs.ac.za
}

\section{Pieter Meintjes}

Physics Department, University of the Free State, 9300, South Africa

E-mail: meintpjegmail.com

\section{Sarah Buchner}

SKA, The Park, Park Road, Pinelands, 7405, South Africa

E-mail: sbuchnerdska.ac.za

Pulsars are extremely accurate clocks that allow us to explore certain unanswered questions in the fields of nuclear and gravitational wave astrophysics. Pulsars that form part of a timing array can be used to detect stochastic gravitational wave (GW) backgrounds produced by merging supermassive black holes. However, these backgrounds produce a small amplitude variation in the timing residuals of a pulsar over decades. A number of elements influence the accuracy of the observed times of arrival (TOA); of which "timing noise" contributes to the noise budget due to elements of the pulsar model that we do not understand, as well as the dispersive nature of the ISM through which the pulse propagates. We analyze two decades of data of the pulsar PSR J1326-5859 to observe possible magnetospheric switches that influence the pulsar spin-down which could lead to a deeper understanding of the timing noise phenomena. Here we present the necessary analysis pipeline to investigate the long term stability of the pulsar timing noise, which may lead us to a better understanding of all the contributing factors influencing timing noise. This may then allow possible identification of GW signatures in the pulsar timing noise data.

Frontier Research in Astrophysics,

26-31 May 2014

Mondello (Palermo), Italy

* Speaker.

${ }^{\dagger}$ The speaker wishes to acknowledge the SKA for financial support. 


\section{Introduction}

Pulsars are rapidly rotating, highly magnetized objects that are the remnants from supernovae explosions. These objects contribute greatly to the fields of astrophysics and nuclear physics due to its peculiar high core density and fast spin-periods that range from milliseconds to several seconds (e.g. [1]). However, not all pulsars are considered stable clocks. Pulsar timing represents the technique of comparing the observed and computed pulse times of arrival (TOA), this comparison produces a residual that is non-zero and is more commonly known as timing noise. Timing noise varies as a quasi-periodic signature over times-scales of decades.

Together with GW background timing perturbations, these form part of the pulsar red noise reservoir. GW stochastic backgrounds perturb the spacing between the emitted pulses on a scale of $\Delta L / L \approx 10^{-14}$. The timing residual signature of a GW background scales as a power law of the GW frequency, this is $h \approx A_{g}\left(\frac{f_{g}}{y r^{-1}}\right)^{\alpha}$, with $A_{g}, f_{g}$ and $\alpha$ being the amplitude of GW background, the frequency of the GW background (in units of years ${ }^{-1}$ ) and the spectral index. The size of the residuals induced by GW and timing noise are in the order of microseconds and possibly seconds, respectively. The effect can be simulated on a set of pulsars (with known ephemerides) using the Tempo2 code ([2]).

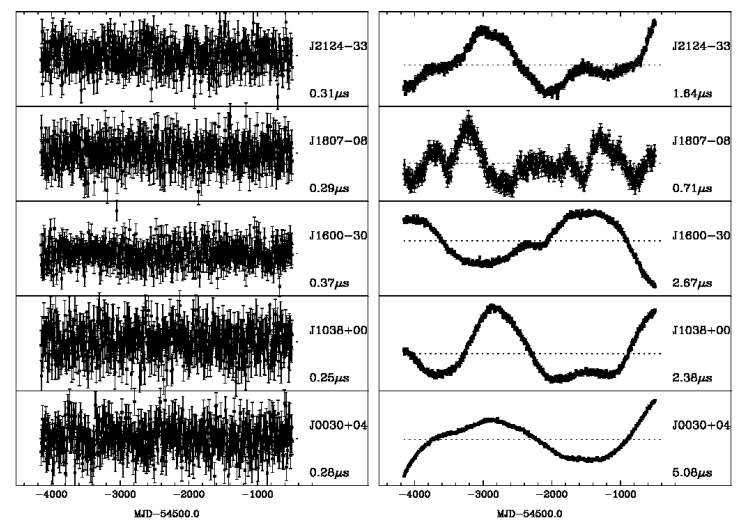

Figure 1: The effect on pulsar timing residuals of GW produced by merging SMBHs(e.g. [1] and [3]).

Detection and characterization of this subtle residual signature depends on deeper insights into other elements that influence the TOA precision. [4] showed the existence of switched magnetospheric regulation of pulsar spin-down. This effect can be seen as a variation between several states in the spin-down $(\dot{v})$ and pulse profiles of the pulsar, with time. We propose a similar analysis pipeline on the HartRAO (Hartebeeshoek Radio Astronomical Observatory) observed pulsar PSR J1326-5859, which is a $478 \mathrm{~ms}$ pulsar with an effective pulse width of $90 \mathrm{~ms}$. These changes in the pulsar magnetosphere could possibly be linked with timing noise and other pulsar emission phenomena, including pulse-nulling and pulse variations ([4]). Understanding and correcting for timing noise effects in pulsar timing will help us reach the necessary $\mu s$-timing accuracy needed for GWs detection. In this paper we present the methodology of the timing noise analysis pipeline by analyzing only a part of the timing data associated with PSR J1326-5859. 


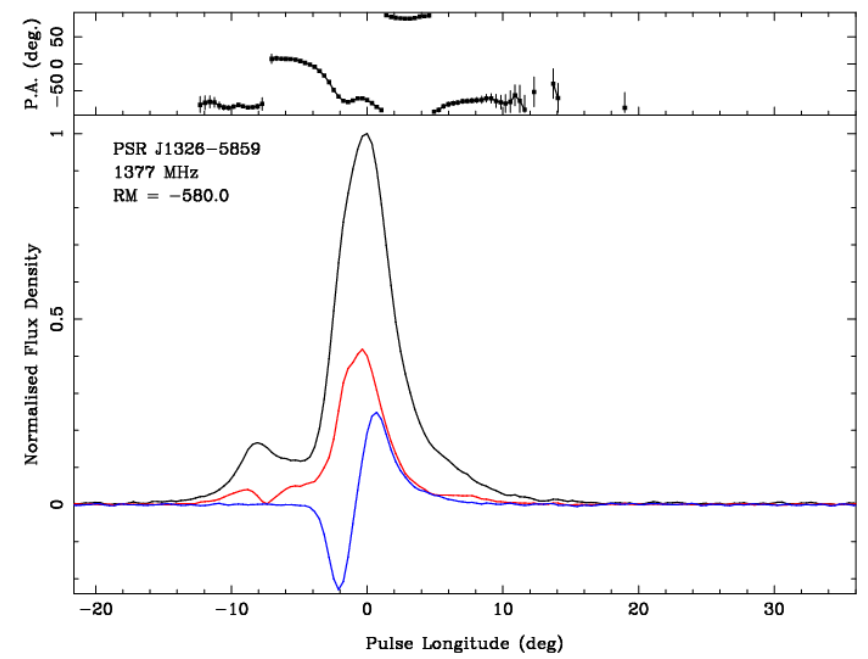

Figure 2: Standard Pulse profile of PSR J1326-5859, from www.atnf.csiro.au ([5]). This includes the Stokes profiles that can also be used in the timing analysis.

\section{Timing pulsars}

When timing pulsars, the element of interest is the times of arrival (TOA). Pulsars experience a spin-down due to the loss of energy through electromagnetic radiation processes and relativistic particle winds. We describe the pulsar rotation in a reference frame that is co-moving with the pulsar. The barycentric TOA of the pulse can be modeled by including all the effects that cause a delay in the arrival time of the pulse (e.g. [1]). This is

$$
T O A=t_{\text {topo }}+t_{\text {corr }}-\Delta_{D M}+\Delta_{\text {solar barycenter }}+\Delta_{G W}+\Delta_{\text {timing noise }} .
$$

The difference between the modeled and observed TOAs produces non-zero timing residuals that contain signatures that are intrinsic to the pulsar model or the observational instrument. This process is done through a least-squares fit of the pulsar model to the observed TOA. These signatures include: neglected astrometric elements of the pulsar model, glitches, giant pulses and timing noise (e.g. [1]).

There are many factors that determine the timing precision of a pulsar. Using a first approximation, the general timing precision will be $\sigma_{T O A} \propto W /(S / N)$. Here $W$ and $S / N$ represent the pulse width and the signal to noise ratio of the observed pulse.

The signal to noise ratio is

$$
S / N=\frac{T_{A} \sqrt{B \tau}}{T_{s y s}} .
$$

It is evident from the radiometer equation that a longer integration time $(\tau)$, large observational bandwidth $(B)$ and minimal system temperature (which could be at least a few tens of Kelvins) will produce the needed signal to noise ratio. Here $T_{A}$ is the brightness temperature of the source, with $T_{\text {sys }}=T_{\text {sky }}+T_{\text {receiver }}$. Thus millisecond pulsars, in principle, will be the best candidates for timing 


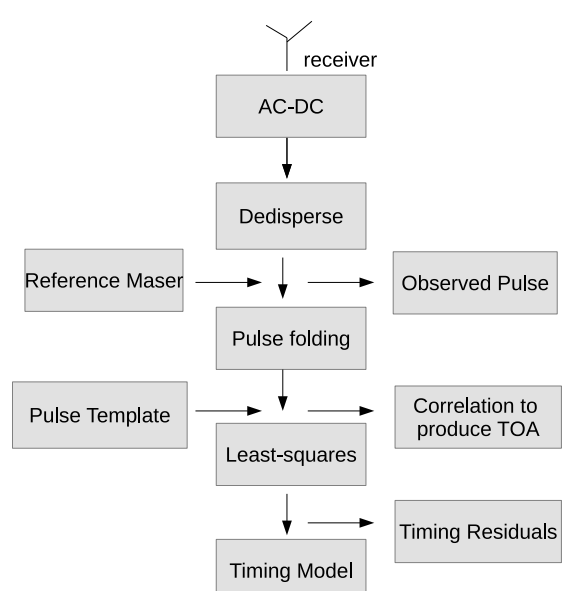

Figure 3: Timing scheme of pulsar observations. The observed signal passes through a filter bank where it is dedispersed and folded (e.g. [1])

precision due to their stable profiles and narrow pulse widths. Timing noise is therefore a fundamental problem, the solution of which will lead to more accurate timing models and techniques to detect stochastic gravitational wave backgrounds.

\section{Timing noise: Analysis Pipeline}

Recent papers propose methods for correlating timing noise with certain changes in pulsar spin-parameters ([4]). We use this method on PSR J1326-5859 that was observed by the $26 \mathrm{~m}$ HartRAO radio telescope for several decades with a weekly cadence at $1668 \mathrm{MHz}$. We analyzed only a part of the data, since further detailed analysis are planned for a future paper.

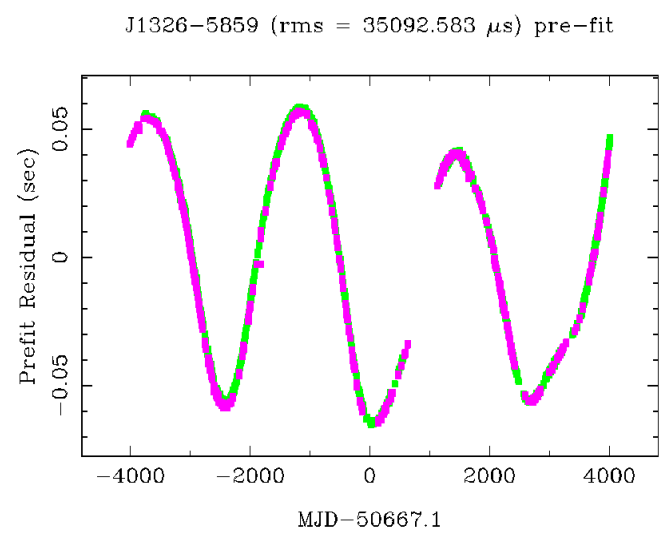

Figure 4: Timing residuals for PSR J1326-5859 produced with the Tempo 2 code. The gaps in the data represent time periods when observations ceased ([2]). The non-zero signature in the timing residual represents timing noise. 
The spin-down evolution was calculated by dividing the total MJD-span into 300-day sections $(\delta t)$. We used the Tempo2 glitch-plugin ([2]) to analyze each section and calculate the spin-down evolution $(\dot{v})$ with time. In this paper we present only part of the analysis, that is for MJD=48000 to $\mathrm{MJD}=51000$. PSR J1326-5859 has a frequency of $2.092 \mathrm{~Hz}$ with a mean spin-down rate of $-1.4 \times$ $10^{-14} \mathrm{~Hz} / \mathrm{s}$ and a fractional change in the spin-down rate of $\approx 2.5 \%$. This switched magnetospheric regulation in the spin-down of PSR J1326-5859 was also observed by [6].

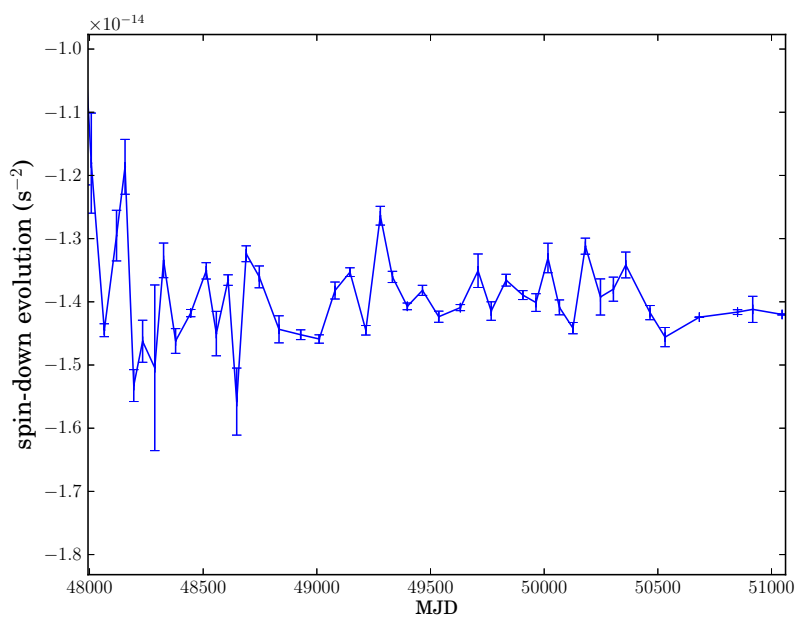

Figure 5: Analysis of the spin parameter $\dot{v}$ for a section of the 21 year data set of PSR J1326-5859.

The last part of the timing noise analysis pipeline addresses the Stokes decomposition of the observed single pulses. Unfortunately we did not manage to record the polarization data, since the pulsar timer associated with the $26 \mathrm{~m}$ HartRAO radio telescope is currently in a testing phase. The vector sum of the electromagnetic waves from the radiating source at the antenna of the telescope has a net polarization that is generally stated in terms of the Stokes parameter $I, Q, U$ and $V$. These parameters are related, that is $I^{2}=Q^{2}+V^{2}+U^{2}$. These parameters are generated in the hardware with the use of a polarimeter. The aim of this analysis is to search for changes in the Stokes parameters over the length of the data set. A purely linearly polarized wave has $V=0$, while $V / Q=$ 1 for right-circular polarized and $V / Q=-1$ for left-circular polarized. The relation $\tan (2 \Phi)=$ $U / Q$ defines the shape of an ellipse. In addition we will also calculate the ratios $V / I$ and $Q / I$ to search for any changes in the linear or circular polarization of the observed radiation. This part of the analysis will hopefully produce correlations between the Stokes profiles and magnetospheric switches, as well as providing a diagnostic tool to identify temporal GW signatures.

If each part of the pipeline is fully developed and tested, we will eventually analyze all the HartRAO observed pulsars. This will produce a large data set that will be used to cross-correlate timing noise between the HartRAO pulsars and also relate the observed timing noise with any changes in spin-down parameters and the pulse profiles of the pulsars. We are planning to enable the HartRAO pulsar timer to start recording polarization data for future pulsar observations. 


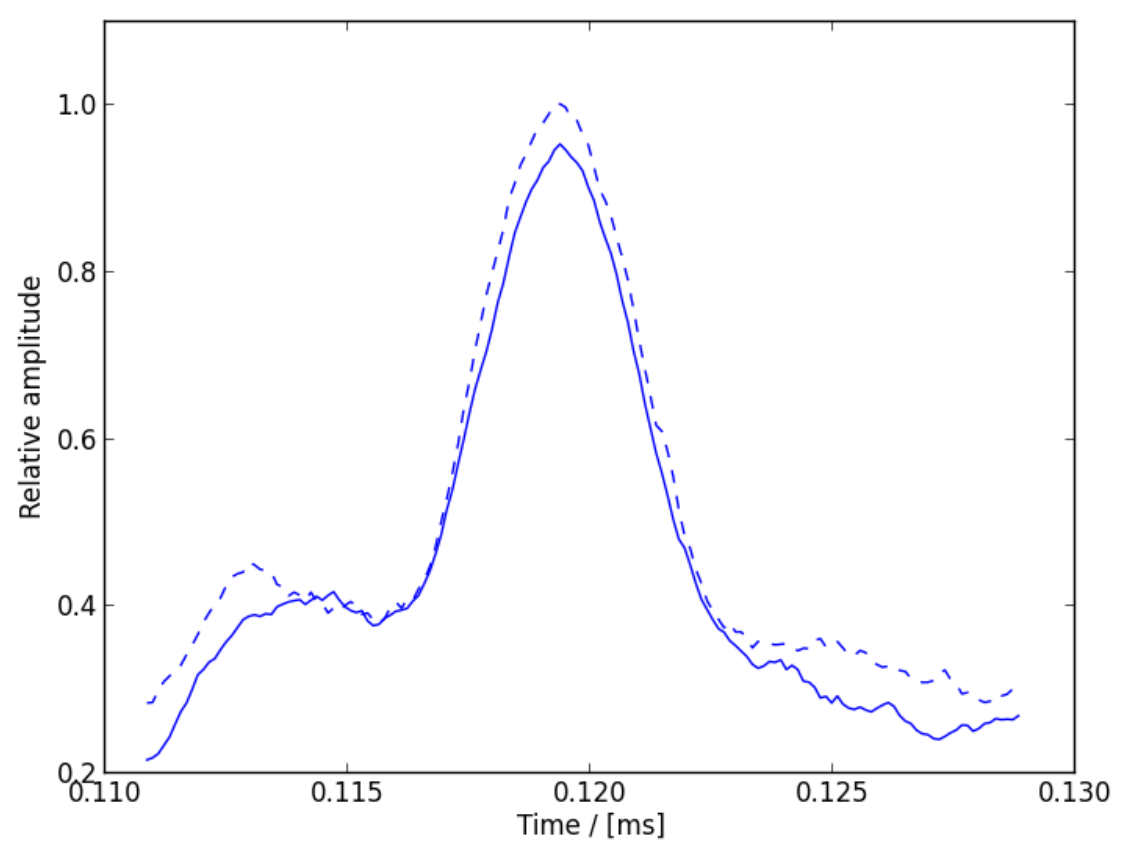

Figure 6: Standard pulses for two observational regions: at MJD $=47750$ (line) and MJD $=49000$ (dashed line). The standard pulse profiles correspond to the standard archival profiles.

\section{Discussion and conclusions}

[6] performed similar timing noise statistical analysis on 27 HartRAO pulsars. The observed fluctuations between states in the spin-down evolution are seen in both Parkes ([4]) and HartRAO pulsar long term data and could be linked with pulse shape variations. Evidently, the pulse profiles at the two extreme spin-down states are different.

The observed structures in the timing residuals of PSR J1326-5859 arise from the spin-down rate switching systematically between states. The two distinct spin-down states correspond with the most extreme pulse shapes observed at the two states (this was for MJD $=48000$ to MJD $=$ 51000). This could possibly be a relationship between the spin-down evolution and magnetospheric switches.

The accuracy and sensitivity of modern day radio telescopes are being improved at an dramatic rate, however magnetospheric state switching is responsible for limiting the accuracy of the timing accuracy. The aim is to model and remove the timing noise and improve the prospects of producing an essentially stable clock. The future of timing noise analysis will depend on high resolution single pulse data observed with low-noise, sensitive radio telescopes.

\section{References}

[1] D. R. Lorimer. Handbook of pulsar astronomy. Cambridge University Press, Cambridge, UK New York, 2005. ISBN 978-0-521-82823-9. 
[2] G. Hobbs, R. Edwards, and R. Manchester. TEMPO2: a New Pulsar Timing Package. CJAA, 6(2):020000-192, December 2006.

[3] G. Hobbs, F. Jenet, K. J. Lee, J. P. W. Verbiest, D. Yardley, R. Manchester, A. Lommen, W. Coles, R. Edwards, and C. Shettigara. TEMPO2: a new pulsar timing package - III. Gravitational wave simulation. MNRAS, 394:1945-1955, April 2009.

[4] A. Lyne, G. Hobbs, M. Kramer, I. Stairs, and B. Stappers. Switched Magnetospheric Regulation of Pulsar Spin-Down. Science, 329:408-, July 2010.

[5] R. N. Manchester, G. B. Hobbs, A. Teoh, and M. Hobbs. VizieR Online Data Catalog: ATNF Pulsar Catalog. VizieR Online Data Catalog, 7245:0, August 2005.

[6] A. E. Chukwude. A Statistical Analysis of Radio Pulsar Timing Noise. CJAA, 7:521-530, August 2007. 